\title{
A Personalized Recommendation Algorithm Based on the User's Implicit Feedback in E-Commerce
}

\author{
Bo Wang *, Feiyue Ye and Jialu Xu \\ School of Computer Engineering and Science, Shanghai University, Shanghai 200444, China; \\ yefy@shu.edu.cn (F.Y.); jialuxu@shu.edu.cn (J.X.) \\ * Correspondence: longxinwu@shu.edu.cn; Tel.: +86-131-6711-9225
}

Received: 28 October 2018; Accepted: 27 November 2018; Published: 29 November 2018

\begin{abstract}
A recommendation system can recommend items of interest to users. However, due to the scarcity of user rating data and the similarity of single ratings, the accuracy of traditional collaborative filtering algorithms (CF) is limited. Compared with user rating data, the user's behavior $\log$ is easier to obtain and contains a large amount of implicit feedback information, such as the purchase behavior, comparison behavior, and sequences of items (item-sequences). In this paper, we proposed a personalized recommendation algorithm based on a user's implicit feedback (BUIF). BUIF considers not only the user's purchase behavior but also the user's comparison behavior and item-sequences. We extracted the purchase behavior, comparison behavior, and item-sequences from the user's behavior log; calculated the user's similarity by purchase behavior and comparison behavior; and extended word-embedding to item-embedding to obtain the item's similarity. Based on the above method, we built a secondary reordering model to generate the recommendation results for users. The results of the experiment on the JData dataset show that our algorithm shows better improvement in regard to recommendation accuracy over other $\mathrm{CF}$ algorithms.
\end{abstract}

Keywords: collaborative filtering; comparison behavior; item-pairs; item-embedding; secondary-reordering

\section{Introduction}

With the rapid development of the Internet and the emergence of big data, information and data have exploded in size, and it is more difficult for people to obtain accurate and efficient information in time. Therefore, recommendation systems $[1,2]$ have received more and more attention, including content-based recommendation algorithms [3,4], collaborative filtering algorithms [5-7], and hybrid approaches [8,9]. A good recommendation algorithm can better understand the user's purchase intention and can improve the user's viscosity for the e-commerce platform, thereby increasing the user's purchase rate. Among these algorithms, the collaborative filtering algorithm is widely used in various recommendation systems, such as Amazon, Netflix, and others. The reason is that it has no special requirements on the recommended items and can achieve significant recommendation results.

The nearest neighbors approach (KNN) and matrix factorization (MF) are major collaborative filtering techniques. Most of them are designed with explicit feedback data such as rating data, which needs the recommendation systems to guide the users in terms of score. However, users are often reluctant to spend time reviewing items in the field of e-commerce, which causes the rating data to be difficult to obtain and sparse.

Some recommendation algorithms are based on user's behavior logs and can solve the difficulty of data acquisition. However, the user's behavior logs lack negative feedback-we cannot be directly aware of user's preferences, which makes it more difficult to find the nearest neighbors accurately. In order to solve this problem, we fully mined the consumer's implicit feedback, such as the purchase behavior, comparison behavior, and item-sequences. The TF-IDF (Term Frequency-Inverse Document 
Frequency) algorithm and the word-embedding technology of the natural language processing (NLP) field were improved and applied to the collaborative filtering algorithm. The contributions of our work are as follows:

- Refine the user's behavior, build item-pairs, and consider not only the user's purchase behavior but also the comparison behavior, and apply TF-IDF to collaborative filtering (CF) to calculate the user's similarity more reasonably.

- Extract the user's browse sequence for items from the user's behavior log, use word-embedding technology to map items to low-latitude vector spaces, and calculate the similarity of the items.

- Construct a secondary reordering model to generate the recommendation results for the users.

\section{Related Work}

According to the classification method proposed by Breese [10], CF algorithms can be divided into two categories: memory-based CF, which includes information based on the user's CF [11,12] and item CF [13]; and model-based CF [14]. Traditional collaborative filtering algorithms are mostly based on explicit data, such as ratings or stars, which is sparse in e-commerce. Therefore, more and more scholars have studied the user's implicit feedback to recommend items to users.

$\mathrm{Hu}$ [15] treated the data as an indication of positive and negative and tailored a factor model for implicit feedback to recommend television shows. Choi [8] considered only the purchase data of the user to derive implicit ratings, to enable $\mathrm{CF}$ to be applied, and then they integrated $\mathrm{CF}$ and (sequence pattern analysis) SPA to improve the recommendation quality. Rendle [16] directly optimized for ranking by Bayesian personalized ranking (BPR). In Reference [17], the authors incorporated visual features with BPR for the task of personalized ranking. Li [18] trained multiple GBDT (Gradient Boosting Decision Tree) models with a user's characteristics extracted from the user's behavior $\log$, and the output was regarded as the input for LR (Logistic Regression) to obtain the final recommendation results. Reference [19] analyzed the user's shopping log with SVD++ to obtain the user's brand preference. Zhao [6] studied CF in an interactive setting. They used a probabilistic matrix factorization model to capture the distributions of the users and item feature vectors. Barka [20] produced embedding for items in a latent space to analyze item-item relations to produce item similarities. Ozsoy [21] applied Word2Vec to a recommendation system to learn a latent representation of the users and items, and then combined a collaborative filtering algorithm to recommend items to the user.

Compared with the above methods, in this paper, we mined not only the user's purchase behavior but also comparison behavior and item-sequences to recommend items to users. We combined the user's purchase behavior and comparison behavior to modify the method of measuring the user's similarity. The purchase behavior was easy to capture, but not the comparison behavior. Thus, we built item pairs to capture the user's comparison behavior to obtain the user's preferences. In addition to this step, we extracted the item sequences based on the user's behavior log and calculated the items' similarities through an item-embedding technique. Lastly, we built a secondary reordering model to recommend items to users. The results of our method on the JData dataset were compared with other recommendation algorithms, and our method obtained better performance.

\section{User's Similarity Calculation by Combining User's Purchase Behavior and Comparison Behavior}

Due to the lack of rating information in the user's behavior log, the user's similarities cannot be directly calculated. Therefore, in this part, we considered the user's purchase behavior and comparison behavior to measure the user's similarity. By analyzing the user's purchase behavior, we can directly know the user's love for the item. However, in the user's behavior log, there are many other behaviors, such as clicking behavior, adding item to shopping cart behavior, and collecting behavior. When a user wants to buy an item in JD (a large e-commerce platform in China), the different behaviors reflect 
different degrees of user interest for the item. Additionally, if two users select the same item when facing the same two items, they have similar comparison behavior and are more similar in preference.

\subsection{User's Browsing Behavior Analysis}

In e-commerce, a user's behavior is divided into clicking, add item to shopping cart (represented by adding in the following), collecting, and buying. We defined the user's behavior as $\mathrm{B}=\left(b_{1}, b_{2}, b_{3}, b_{4}\right)$ where $b_{1}$ means clicking, $b_{2}$ means adding, $b_{3}$ means collecting, and $b_{4}$ means buying. Intuitively, in the user's behavior log, a certain behavior occurs more with lower interest, and otherwise with higher interest. Table 1 shows the user's behavior-matrix extracted from the user's behavior log. Here, $f_{k}$ describes a type of feedback behavior, and $n_{i, k}$ describes the average number of $f_{k}$ for user $i$. Then, we obtained the user's behavioral-habit matrix, as shown in Table 2, by Equation (1):

$$
w_{f_{k}}=\frac{\sum_{k}^{4} n_{f_{k}} / n_{f_{k}}}{\sum_{j}^{4} \sum_{k}^{4} n_{f_{k}} / n_{f_{j}}}
$$

where $n_{f_{k}}$ describes the average number of $f_{k}$, and $w_{f_{k}}$ describes the weight of $f_{k}$. Here, $w_{i, k}$ describes the weight of the user's different feedback in Table 2.

Table 1. User's behavior-matrix.

\begin{tabular}{ccccc}
\hline User & $f_{1}$ & $f_{2}$ & $f_{3}$ & $f_{4}$ \\
\hline $\boldsymbol{u}_{1}$ & $n_{1,1}$ & $n_{1,2}$ & $n_{1,3}$ & $n_{1,4}$ \\
$\boldsymbol{u}_{2}$ & $n_{2,1}$ & $n_{2,2}$ & $n_{2,3}$ & $n_{2,4}$ \\
$\ldots$ & $\ldots$ & $\ldots$ & $\ldots$ & $\ldots$ \\
$\boldsymbol{u}_{\mathbf{i}}$ & $n_{i, 1}$ & $n_{i, 2}$ & $n_{i, 3}$ & $n_{i, 4}$ \\
\hline
\end{tabular}

Table 2. User's behavior-habits-matrix.

\begin{tabular}{ccccc}
\hline User & $f_{1}$ & $f_{2}$ & $f_{3}$ & $f_{4}$ \\
\hline $\boldsymbol{u}_{1}$ & $w_{1,1}$ & $w_{1,2}$ & $w_{1,3}$ & $w_{1,4}$ \\
$\boldsymbol{u}_{2}$ & $w_{2,1}$ & $w_{2,2}$ & $w_{2,3}$ & $w_{2,4}$ \\
$\ldots$ & $\cdots$ & $\cdots$ & $\cdots$ & $\cdots$ \\
$\boldsymbol{u}_{\mathbf{i}}$ & $w_{i, 1}$ & $w_{i, 2}$ & $w_{i, 3}$ & $w_{i, 4}$ \\
\hline
\end{tabular}

Unlike the explicit feedback, we do not have any direct data from the users with regard to their preferences, but we can extract the user's behavior-habit instead, which is represented by $W_{u}=\left(w_{u, 1}, w_{u, 2}, w_{u, 3}, w_{u, 4}\right)$. Otherwise, we analyzed the user's behavior log and build the user's interaction vector for items that are represented by $n_{i}=\left(n_{1}, n_{2}, n_{3}, n_{4}\right)$. Therefore, we obtained the user's preferences for items based on the user's interaction with the recommendation system by Equation (2):

$$
\text { Score }_{u, i}=w_{u} * n_{i},
$$

where $\operatorname{Score}_{u, i}$ represents the user's preference for items.

\subsection{User's Comparison Behavior Analysis}

When purchasing items, users will compare a series of items and finally purchase one. The process of selecting items reflects the user's comparison behavior. If users have similar comparison behaviors, they should be more similar. In this section, we built item pairs to capture a user's comparison behavior and obtain a user's real preference for items.

Definition 1. Purchase cycle T. In real life, users often buy items on an e-commerce platform for a period of time. Therefore, we split the user's behavior log into different purchase cycles based on the time when the user's purchase behavior occurs, $T=\left(t_{1}, t_{2}, \ldots, t_{n}\right)$. 
Definition 2. Item-pair $\boldsymbol{p}_{(u, i>j)}$. This concept describes a user's comparison behavior before purchasing items in a purchase cycle, and it can be used to measure the user's similarity.

Table 3 is an example to show how to build an item-pair.

Table 3. A series of feedback for $u_{1}$ in a purchase cycle.

\begin{tabular}{ccccc}
\hline User & Interaction Log & Adding & Collecting & Buying \\
\hline$u_{1}$ & $a, a, b, a, c, a, a$ & $a, c$ & $a$ & $a$ \\
\hline
\end{tabular}

Table 3 shows the user's interaction vectors to items represented by $n_{u_{1}, a}=(2,1,1,1)$, $n_{u_{1}, b}=(1,0,0,0)$, and $n_{u_{1}, c}=(2,1,0,0)$. The user's behavior-habits matrix has been obtained in "Section 3.1", and thus, the preference to items of $u_{1}$ can be obtained by Equation (2). Then we can build the item-pairs $P_{\left(u_{1}, a>b\right)}, P_{\left(u_{1}, a>c\right)}$, and $P_{\left(u_{1}, c>b\right)}$.

\subsection{User's Similarity Calculation}

Each user has their own item-pair sets, and different item-pairs have different weights for the user, to calculate user's similarity. An example is shown in Table 4.

Table 4. User's item-pairs sets.

\begin{tabular}{cc}
\hline User & Item-Pairs Set \\
\hline$u_{1}$ & $P_{\left(u_{1}, a>b\right)}, P_{\left(u_{1}, a>c\right)}, P_{\left(u_{1}, c>b\right)}, P_{\left(u_{1}, c>d\right)} \ldots \ldots$ \\
$u_{2}$ & $P_{\left(u_{2}, a>b\right)}, P_{\left(u_{2}, c>a\right)}, P_{\left(u_{2}, c>b\right)}, P_{\left(u_{2}, d>c\right)} \ldots \ldots$ \\
$u_{3}$ & $P_{\left(u_{3}, a>b\right)}, P_{\left(u_{3}, c>a\right)}, P_{\left(u_{3}, c>b\right)}, P_{\left(u_{3}, d>c\right)} \ldots \ldots$ \\
$u_{4}$ & $P_{\left(u_{4}, a>b\right)}, P_{\left(u_{4}, a>c\right)}, P_{\left(u_{4}, c>b\right)}, P_{\left(u_{4} c>d\right)} \ldots \ldots$ \\
$u_{5}$ & $P_{\left(u_{5}, a>b\right)}, P_{\left(u_{5}, a>c\right)}, P_{\left(u_{5}, c>b\right)}, P_{\left(u_{5}, c>d\right)} \ldots \ldots$ \\
\hline
\end{tabular}

When facing $a$ and $c$, three-fifths of consumers choose $a$, and two-fifths choose $c$, and thus, $P_{(u, c>a)}$ should be a greater weight for $u_{2}, u_{3}$ than for $u_{1}, u_{4}, u_{5}$.

In the field of natural language processing (NLP), the TF-IDF algorithm is a statistical method to assess the importance of the same word for one file or one corpus. The corpus is similar for user groups. Therefore, in this paper, we tried to apply TF-IDF to a recommendation algorithm. We regarded the user groups as a corpus, the user's item-pair sets as one file, and an item-pair as a word.

Definition 3. Item-pair Frequency (IPF). This concept describes the times that an item-pair appears in the item-pair sets of a user. The larger the number of times is, the more important the item-pair is to the user.

Definition 4. Inverse item-pair Frequency (IIPF). This concept describes how many user's item-pair sets contain the item-pair. The larger the number of times that an item-pair appears in all user's item-pair sets, the worse the item-pair is for distinguishing the user when calculating the user's similarity.

Figure 1 shows the process of IPF-IIPF, and the weight of the item-pair for each user can be calculated by Equation (3):

$$
I P F-\operatorname{IIPF} F_{w\left(p_{i}, u_{j}\right)}=\operatorname{IPF}_{(i, j)} \times \operatorname{IIPSF} F_{(i, j)}=\frac{n_{p_{i}, u_{j}}}{\sum_{k} n_{p_{k}, u_{j}}} \times \log \left(\frac{|U|}{1+\left|j: p_{i} \in u_{j}\right|}\right),
$$

where $p_{i}$ means an item-pair, $u_{j}$ means a user, $n_{p_{i}, u_{j}}$ means the number of occurrences of $p_{i}$ in the item-pair set of $u_{j}$, and $|U|$ means all users. 
Then, the user's similarity can be calculated by Equation (4):

$$
\operatorname{sim}_{\text {pair_item }}(u, v)=\frac{\sum_{p_{j} \in I P_{u, v}}^{k}\left(w_{u, p_{j}}-\overline{w_{u}}\right)\left(w_{v, p_{j}}-\overline{w_{v}}\right)}{1+\sqrt{\sum_{p_{i} \in I_{u}}^{n}\left(w_{u, p_{i}}-\overline{w_{u}}\right)^{2}} \sum_{p_{j} \in I_{v}}^{n}\left(w_{v, p_{j}}-\overline{w_{v}}\right)^{2}},
$$

where $I P_{u, v}$ means the common item-pairs of user $u$ and user $v, p_{i}, p_{j}$ means the item-pair, and $\overline{w_{u}}$ means the average weight of the user's item-pairs.

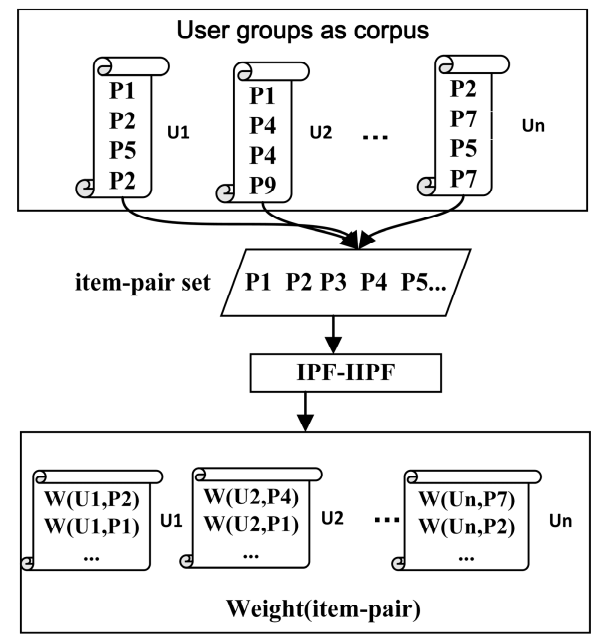

Figure 1. Overview of IPF-IIPF (Item-pair Frequency—Inverse item-pair Frequency).

\section{Item's Similarity Calculation Based on Item Embedding}

In the user's behavior log, the user's interaction record over time contains a large amount of information on the items. For example, if a user wants to buy a computer, they will compare a series of computers, and the compared sequence shows a high degree of similarity between items. To calculate the similarity of these items, we applied word-embedding technology to train item-sequences to obtain item vectors and measure the item's similarity.

Word2vec is a group of models of word-embedding technologies, which was proposed by Mikolov [22,23]. It contains two different models, namely Skip-gram and CBOW (Continuous Bag-of-Words), which use either hierarchical softmax or negative sampling. Skip-gram and CBOW models are all shallow three-layer neural network models, and in contrast to traditional DNN (Deep Neural Networks) models, they use a Huffman tree to replace neurons in the hidden and output layers, which reduced the calculations. In this section, we used both of the models to process the sequence of items that were browsed by a user.

\subsection{Extract the Item-Sequences}

Applying word2vec technology to a recommendation algorithm [24], we must first obtain training data that is similar to the corpus, which is composed of sentences.

We split the user's behavior log into different purchase cycles by the algorithm shown in Algorithm 1 and extracted the item-sequences shown in Table 5. In each cycle, only one item is purchased by the user. 
Table 5. Item-sequences.

\begin{tabular}{|c|c|}
\hline$T$ & Item-Sequence \\
\hline$T_{1}$ & item $_{1}$, item $_{3}$, item $_{3}$, item $_{4} \ldots$ \\
\hline$T_{2}$ & item $_{2}$, item $_{6}$, item $_{4}$, ,tem $_{9} \ldots$ \\
\hline$T_{3}$ & item $_{3}$, item $_{6}$, item $_{8}$, item $_{10} \ldots$ \\
\hline$T_{4}$ & item $_{4}$, item $_{12}$, item $_{12}$, item $10 \ldots$ \\
\hline$\ldots$ & $\ldots \ldots$ \\
\hline
\end{tabular}

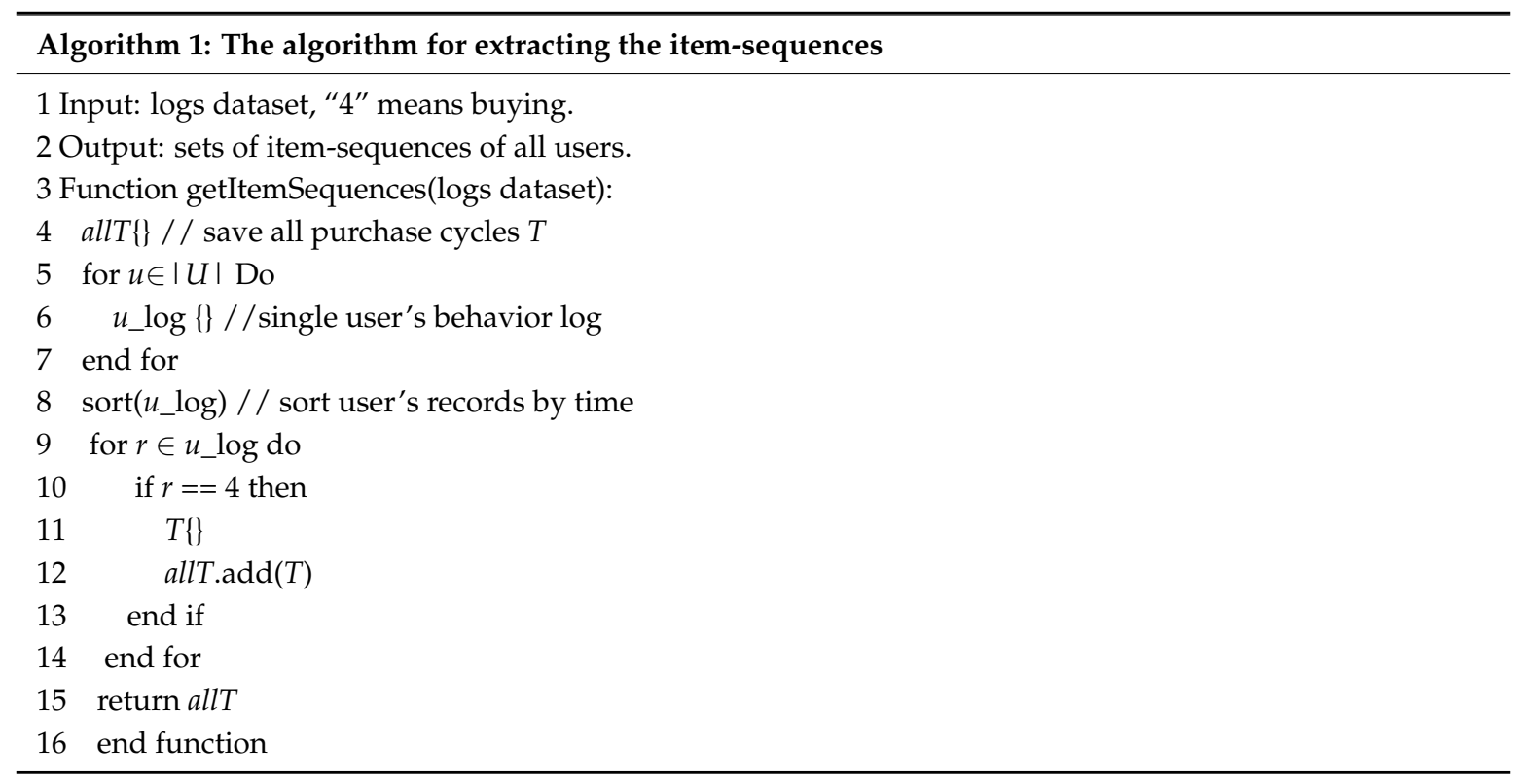

\subsection{Item Vector Representation and Similarity Calculation}

In this part, we applied the word2vec to train the item-sequences and obtain the item vectors. The main models of word2vec are CBOW and Skip-gram, with the input a large corpus of sentences. Word2vec builds a dictionary as an input to the training models, which is a three-layer neural network for words that appear in the corpus.

The CBOW predicts the target word from the original sentence. We entered the words $w_{t-2}, w_{t-1}, w_{t+1}, w_{t+2}$ around the target word $w_{t}$ and maximized the likelihood function, as shown in Equation (5), by the stochastic gradient descent algorithm, to obtain a vector of the target word. The Skip-gram is the opposite. Figure 2 shows the difference of the two models.

$$
\zeta=\sum_{w \in \mathcal{c}} \log p\left(w_{t} \mid \operatorname{context}\left(w_{t}\right)\right),
$$

The process of recommendation is similar to the process of CBOW and Skip-gram to predict vectors of words, and therefore, we regarded items as words and extracted item-sequences from the user's behavior log as sentences. Then, we put these data into the CBOW model and Skip-gram model to obtain the item's vectors, and we calculated the similarity of the items by Equation (6):

$$
\operatorname{sim}(i, j)=\frac{V_{i} * V_{j}}{\left\|V_{i}\right\|\left\|V_{j}\right\|^{\prime}},
$$

where $V_{i}, V_{j}$ means the vectors of item $i$ and $j$. Then, we proposed two algorithms called CBOW-CF and Skip-CF. In the experiment, we have discussed the different influences of the different dimensions of vectors on the recommendation results. 


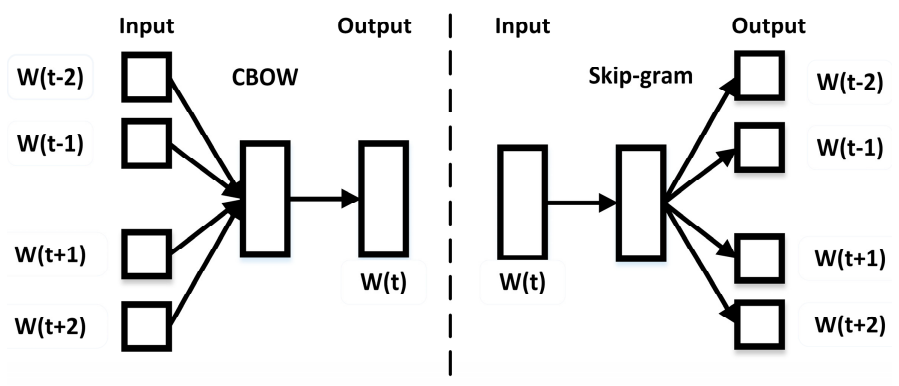

Figure 2. Word2vec models.

\section{The Recommendation Algorithm Based on the User's Implicit Feedback}

Based on the previous introduction, we considered the user's clicking, adding, collecting, and buying behaviors and built item pairs by the user's interaction with the JD system to calculate the user's similarity more accurately, and we learned the vector space representation of items with word2vec technology to calculate the items' similarity more reasonably. Additionally, we obtained the user's candidate set and item's candidate set and relied on the two methods, and then, the recommended results were generated by the secondary reordering. Figure 3 shows the overview of the whole algorithm.

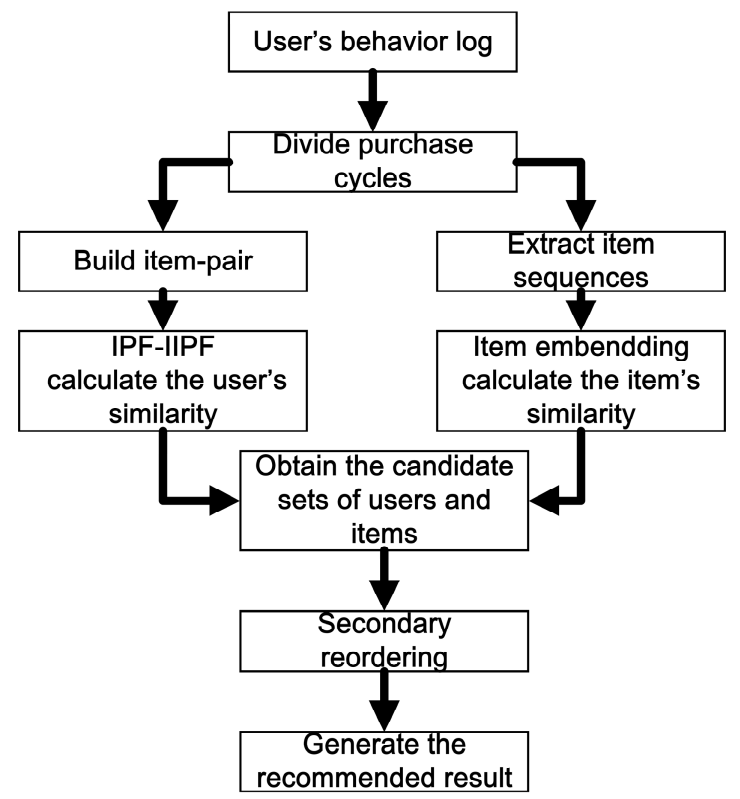

Figure 3. Overview of the recommendation algorithm based on the user's implicit feedback.

When recommending items to users, we should first choose the $\mathrm{K}$ nearest neighbors of the users. Traditional CF always chooses the $\mathrm{K}$ nearest neighbors from all of the users, which increases the calculation time. To solve this problem, we set a threshold $\beta$ to obtain a candidate set of users. Here, $\beta$ is repressed by Equation (7):

$$
\beta=\overline{\operatorname{Sim}_{\text {user }}(u)}=\frac{\sum_{i}^{m} \operatorname{Sim}_{u s e r}(i)}{m},
$$

where $\overline{\operatorname{Sim}_{u s e r}(u)}$ is the average similarity of the target user. If $\operatorname{Sim}(u, v) \geq \beta$, then user $v$ will be added to the candidate set of user $u$.

Different from the method in Reference [18], which calculates the item's similarity by a single item vector, we considered the item-sequences that users had before purchasing a specific item, and we 
attempted to predict the user's purchase intention, as represented by $v e c_{u}$, which is expressed by Equation (8):

$$
\operatorname{vec}_{u}=\frac{\sum_{i}^{n} \operatorname{vec}_{i} * w_{u, k}}{n}
$$

where $v e c_{i}$ indicates the vector of the item that the user had browsed recently, and $w_{u, k}$ indicates the weight of $f_{k}$ of the user.

The same as with a user, we also set a threshold $\gamma$ to obtain a candidate set of items. Here, $\overline{\operatorname{Sim}}_{\text {item }}(i)$ is the average similarity to the user's purchase intention $v e c_{u}$, as expressed by Equation (9):

$$
\gamma=\overline{\operatorname{Sim}_{\text {item }}(i)}=\frac{\sum_{k=1}^{m} \operatorname{Sim}_{\text {item }}(k)}{m}
$$

If $\operatorname{Sim}\left(v e c_{u}, j\right) \geq \gamma$, then item $j$ will be added to the candidate set of $v e c_{u}$.

Reference [3] has proved that the CF based on the items is superior to the CF based on the users. Therefore, we focused on selecting the item's nearest neighbors for recommendations. Equation (10) expresses the traditional CF based on the items.

$$
R_{(u, i)}=\frac{\sum_{j \in \text { set }_{i}} \operatorname{sim}(i, j) \times R_{(u, j)}}{\sum_{j \in \text { set }_{i}}|\operatorname{sim}(i, j)|} .
$$

We obtained $R_{(u, i)}$, which means the preference of user $u$ to item $i$, and then, recommended the items by $R_{(u, i)}$ from high to low.

In contrast to the traditional CF, we considered the user's candidate set to reorder the item's candidate set to obtain better recommendation results. Suppose that $S_{i, j}$ describes the item-support of items $i$ and $j$, respectively; then, it is calculated by Equation (11):

$$
S_{i, j}=\frac{\sum_{v \in \operatorname{Set}_{(u)}}^{n} G(v, i, j)}{\max \left(\sum_{v \in \operatorname{Set}_{(u)}}^{n} G(v, i, j)\right)},
$$

where $i$ and $j$ means items, and $v_{\log }$ means the behavior $\log$ of user $v$. If $i \in v_{\log }, j \in v_{\log }$, then $G(v, i, j)=1$; otherwise, $G(v, i, j)=0$. Therefore, we calculated the $R_{(u, i)}$ more accurately by Equation (12):

$$
R_{(u, i)}=\frac{\sum_{j \in \operatorname{Set}_{(i)}} \operatorname{sim}(i, j) \times R_{(u, j)} \times S_{i, j}}{\sum_{j \in \text { set }_{i}}|\operatorname{sim}(i, j)|} .
$$

We illustrate the specific principle with an example: assume that the candidate set of user $u$ is $\left\{v_{1}, v_{2}, v_{3}, v_{4}\right\}$, and the candidate set of item $i$ is $\left\{j_{1}, j_{2}, j_{3}, j_{4}\right\}$.

Analyzing the behavior logs of $v_{1}, v_{2}, v_{3}, v_{4}$, we obtained:

- $v 1_{\log }=\left\{j_{1}, j_{3}, \ldots\right\}$

- $v 2 \log =\left\{j_{1}, j_{3}, \ldots\right\}$

- $v 3_{\log }=\left\{j_{2}, j_{3}, j_{1} \ldots\right\}$

- $v 4_{\log }=\left\{j_{2}, j_{4}, j_{1} \ldots\right\}$

and $S_{i, j_{1}}=1, S_{i, j_{2}}=0.5, S_{i, j_{3}}=0.75, S_{i, j_{4}}=0.25$. Therefore, we reordered the items' candidate set by Equations (11) and (12) to generate better recommendation results to the users.

\section{Experiments and Analysis}

\subsection{Dataset}

The JData dataset of our experiment was provided by JD, which is the second largest Chinese e-commerce platform. The JData dataset contains user behavior logs from 1 February to 15 April, 
and the time is accurate to the second, which is the main reason why we used the JData dataset. The download site of JData dataset is on the first page. The user's behavior contains four main types: clicking, adding shopping cart, collecting, and buying. In addition, the user's Id, item's Id, precise time of user's behavior, item's category, item's brand, and the type of user's behavior were also included.

First, we preprocessed the data, retaining only the users who had purchased during this period of time. Then, we obtained 6,841,000 behavior records that contained 29,485 users and 21,267 items. We divided these records into training data and testing data. The training data were composed of the records from 1 February to 10 April, and the testing data were composed of the records from 10 April to 15 April. In the training data, there were 6,168,390 behavior records. We divided them into different purchase-cycles and obtained 122,954 purchase-cycles.

\subsection{Evaluation Criterion}

In this paper, our position was to predict the items that users will purchase in the next period by mining the user's behavior log. Therefore, we used precision, recall, and F-score to evaluate the quality of the algorithms.

$$
\begin{gathered}
\text { Precision }=\frac{\mathrm{TP}}{\mathrm{TP}+\mathrm{FP}^{\prime}} \\
\text { Recall }=\frac{\mathrm{TP}}{\mathrm{TP}+\mathrm{FN}}, \\
\mathrm{F}=\frac{2 * \text { Precision } * \text { Recall }}{\text { Precision }+ \text { Recall }}
\end{gathered}
$$

where TP means the set of items in the recommendation results and purchased by the user. FP means the set of items in the recommendation results but not purchased by the user. FN means the set of items that were purchased by user but not in the recommendation results. $F$ is the harmonic average of the precision and recall.

\subsection{Experimental Results and Analysis}

\subsubsection{Experiment 1: Discuss the Methods of Calculating the User's Similarity}

Different methods to measure the similarity of the users can obtain different nearest neighbors, and the recommendation results will be influenced. To illustrate that our method was better, we chose the following methods:

AW: add weight directly to the user's different behaviors. Clicking with 1, adding with 2, collecting with 3 , buying with 5 . Then, we calculated the user's similarity with the Pearson Correlation Coefficient.

CFPP [6]: calculate the user's similarity by the Pearson correlation coefficient with the user's preference, which is computed solely based on the purchase data.

BUPSP (based-user-purchase-comparison-behavior): calculate the user's similarity by the Pearson correlation coefficient with the user's purchase behavior and comparison behavior.

It can be seen that the precision and recall of AW is the worst, from Figure 4 . The reason is that AW does not consider the user's individual preference. The result of CFPP is better than AW but worse than BUPCB, which is because CFPP only considers the user's purchase behavior. Different from the two above methods, BUPCB quantifies the user's different behaviors according to the user's behavior habits and calculates the user's similarity with the user's comparison behavior. In addition, the results prove the importance of considering the user's comparison behavior when calculating the user's similarity. Figure $4 \mathrm{a}$ also shows that the precision is lower when the number recommended increase, which occurs because the test data only include 5 days of JData, and the number of items purchased by a user is small. 


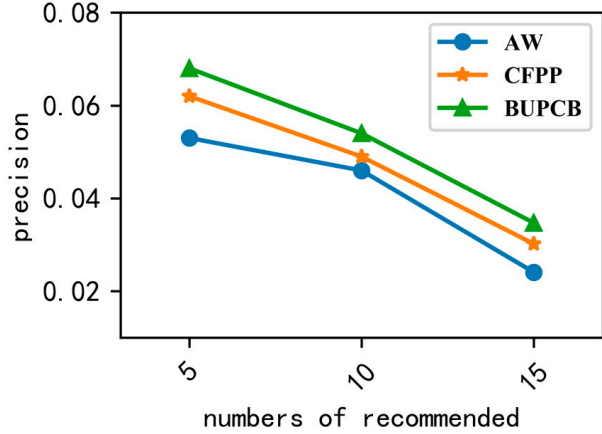

(a)

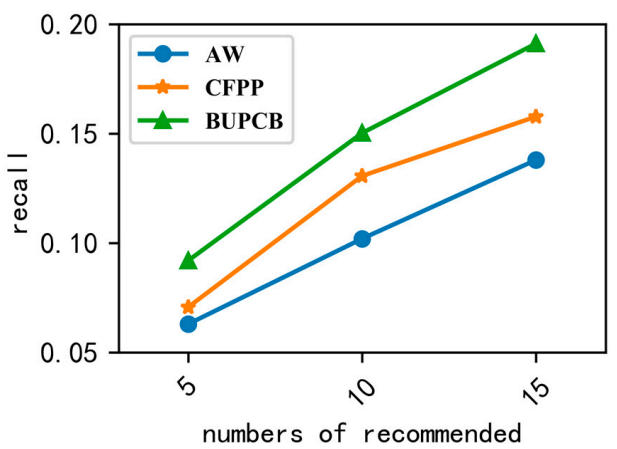

(b)

Figure 4. (a) Describes the precision of the three methods, (b) describes the recall of the three methods.

\subsubsection{Experiment 2: Discuss the Methods of Calculating the Item's Similarity}

In this paper, we used the CBOW model and Skip-gram model to map items to low-latitude vector space, and we used cosine to calculate the item's similarity; the two methods are represented by CBOW-CF and Skip-CF. First, we set the vector size to be from 10 to 70, and obtained the precision shown in Figure 5.

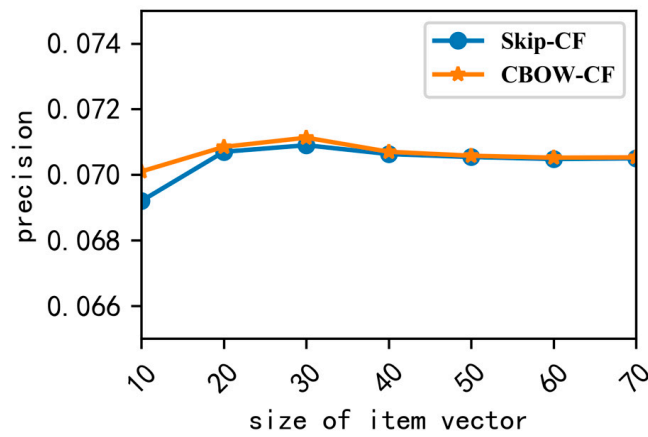

Figure 5. Precision with different vector dimension. CF: collaborative filtering.

Figure 5 shows that when the vector's dimension is 30 , the precision is the best. Therefore, the constant values for the parameters were set to the following: embedding-size $=30$, skip-window $=1$, and train-times $=50,000$, and we removed the items that appeared less than two times.

Figure $6 \mathrm{a}, \mathrm{b}$ shows the comparison with BICF, which calculates the item's similarity by the user's rating, and the interactive times with the items is considered to be the rating data of the user to the items in BICF. The precision and recall of CBOW-CF and Skip-CF, proposed in this paper, were better than BICF, and the result of CBOW-CF was better than Skip-CF. This finding could arise because the training data were small, and the $\mathrm{CBOW}$ model performs better when the training data are small.

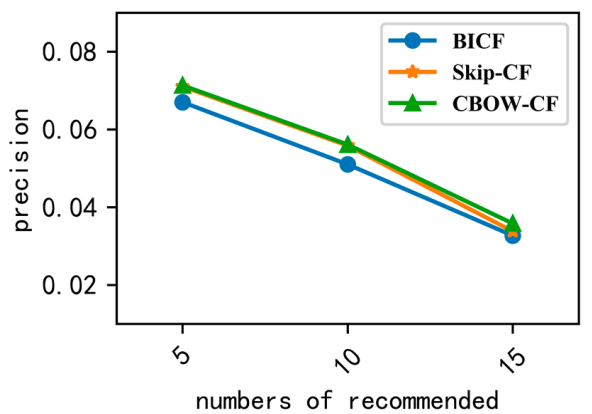

(a)

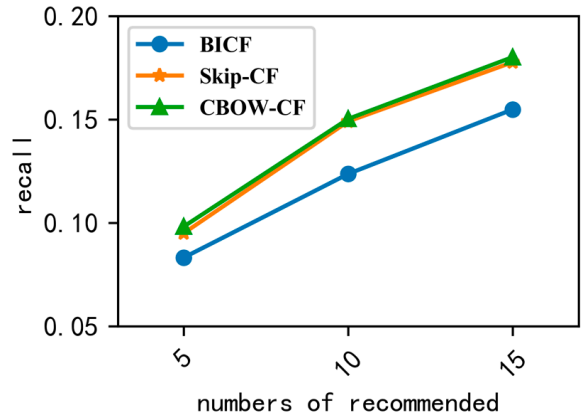

(b)

Figure 6. (a) Describes the precision of the three methods, (b) describes the recall of the three methods. 


\subsubsection{Experiment 3: Comparison with Other Methods}

Because our method was based entirely on the user's implicit feedback, we called it BUIF. To verify the effectiveness of BUIF, the precision, recall, and F1 were compared with the following algorithms while using the same dataset.

BUCF: traditional CF based on users. BUCF calculates the user's similarity by the number of common items that are purchased by the users.

BICF [3]: traditional CF based on items. BICF calculates the item's similarity by the number of common users that purchased the same item.

FPP [6]: builds a rating matrix solely based on the purchase data of the users. FPP integrates CF-based recommendations and SPA-based recommendations.

BUPCB: proposed in this paper, it calculates the user's similarity by the Pearson correlation coefficient with the user's purchase behavior and comparison behavior.

CBOW-CF: trains item-sequences with the CBOW model to obtain the item vectors, and then, combines the traditional $\mathrm{CF}$ to generate the algorithm $\mathrm{CBOW}-\mathrm{CF}$.

BUIF: Our methods described above.

From Figure 7a-c, it can be seen that the precision, recall, and F1 of BUCF and BICF are worse, which is because they regard all behaviors as one type and select the nearest neighbors based on the whole user set and item set. The FPP solely considers the user's purchase data and disregards the information included in clicking, adding, and collecting, which causes the recommendation quality to be reduced. The BUIF method performed better than the others in terms of the precision and recall because BUIF not only considers the user's purchase behavior but also comparison behavior that improved the accuracy of the user's similarity, and BUIF calculates the item's similarity by an item vector that considers the item-sequence information. In addition, the overall accuracy and quality of recommendation were improved effectively through reordering the item's candidate items.

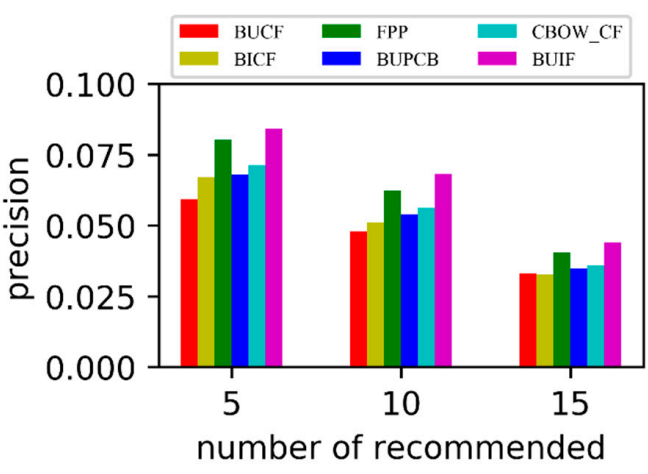

(a)

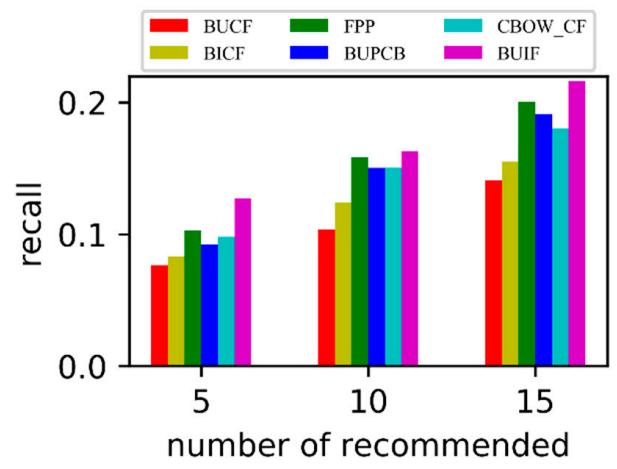

(b)

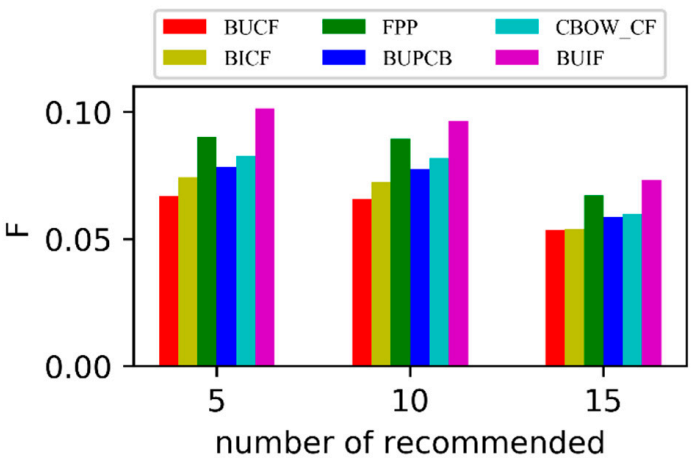

(c)

Figure 7. (a) Describes the precision compared with other methods; (b) describes the recall compared with other methods; (c) describes the F1 compared with other methods. 
There is also an example of a real JD user represented by user_id (290054). We used the above methods to recommend items (number of recommended is five) to the user (290054) and the results are shown in Table 6.

In Table 6, the items are also represented by sku_id. Additionally, the red sku_id means purchased items by the user (290054) in the test data. From Table 5, it can be seen that the recommendation results of BUIF were purchased more than other methods, which means our method will obtain a high degree of user satisfaction.

Table 6. The recommendation results of above methods for user ' 290054 '.

\begin{tabular}{cc}
\hline Methods & Recommend Items \\
\hline BUCF & $78508,109840,44042,1633,143199$ \\
BICF & $118895,15523,120076,70936,170215$ \\
FPP & $135865,130914,109840,57161,43960$ \\
BUPCB & $135272,15523,63231,75750,26229$ \\
CBOW-CF & $160485,109840,24774,62330,170215$ \\
BUIF & $24774,108097,121588,135865,109840$ \\
\hline
\end{tabular}

\section{Conclusions}

This paper proposed a new personalized recommendation algorithm based on the user's implicit feedback. This method fully exploits the implicit information in the user's behavior log, such as the purchase behavior, comparison behavior, and item sequences. Some algorithms in the NLP field, such as TF-IDF and word2vec technologies, were improved and applied to calculate the user's similarity and item's similarity, which make the user's similarity and item's similarity more accurate. Additionally, a secondary reordering screening process was constructed to obtain the final recommended items. From the results, it can be seen that the F1 of BUIF increased an average $28 \%$ and $22 \%$ compared with BUCF and BICF, respectively, when the number of recommended items is 5, 10, and 15. At present, the paper only mines the user's behavior log. In the future, we will combine the user behavior log with the user's demographic features (age, gender, and occupation) and the item's features information (brand, category) and refine it on the basis of a specific group of people or items. In addition, the experiments in this paper were experiments on a part of the data. In future work, we will verify the performance of the algorithm on a larger data set.

Author Contributions: B.W. conceived and designed the experiments; B.W. performed the experiments; B.W., F.Y., and J.X. analyzed the experimental results; B.W. wrote the paper.

Conflicts of Interest: The authors declare no conflict of interest.

\section{References}

1. Burke, R. Hybrid recommender systems: Survey and experiments. User Model. User-Adapt. Interact. 2002, 12, 331-370. [CrossRef]

2. Bobadilla, J.; Ortega, F.; Hernando, A.; Gutiérrez, A. Recommender systems survey. Knowl.-Based Syst. 2013, 46, 109-132. [CrossRef]

3. Guo, W.; Kraines, S.B. Semantic content-based recommendations using semantic graphs. In Advances in Computational Biology; Springer: New York, NY, USA, 2010; Volume 680, pp. 653-659.

4. Musto, C.; Semeraro, G.; Lops, P.; de Gemmis, M. Combining Distributional Semantics and Entity Linking for Context-Aware Content-Based Recommendation. In User Modeling, Adaptation, and Personalization; Springer: Cham, Switzerland, 2014; pp. 381-392.

5. Sarwar, B.; Karypis, G.; Konstan, J.; Riedl, J. Item-based collaborative filtering recommendation algorithms. In Proceedings of the International Conference on World Wide Web, Hong Kong, China, 1-5 May 2001; pp. 285-295. 
6. Zhao, X.; Zhang, W.; Wang, J. Interactive collaborative filtering. In Proceedings of the ACM International Conference on Conference on Information \& Knowledge Management, San Francisco, CA, USA, 27 October-1 November 2013; pp. 1411-1420.

7. Polatidis, N.; Georgiadis, C.K. A dynamic multi-level collaborative filtering method for improved recommendations. Comput. Stand. Interfaces 2017, 51, 14-21. [CrossRef]

8. Choi, K.; Yoo, D.; Kim, G.; Suh, Y. A hybrid online-product recommendation system: Combining implicit rating-based collaborative filtering and sequential pattern analysis. Electron. Commer. Res. Appl. 2012, 11, 309-317. [CrossRef]

9. Cao, J.; Wu, Z.; Zhuang, Y. Hybrid collaborative filtering algorithm for bidirectional web service recommendation. Knowl. Inf. Syst. 2013, 36, 607-627. [CrossRef]

10. Breese, J.S.; Heckerman, D.; Kadie, C. Empirical analysis of predictive algorithms for collaborative filtering. In Proceedings of the Fourteenth Conference on Uncertainty in Artificial Intelligence, Madison, WI, USA, 24-26 July 1998; Morgan Kaufmann Publishers Inc.: San Francisco, CA, USA, 1998.

11. Ye, F.; Zhang, H. A collaborative filtering recommendation based on users' interest and correlation of items. In Proceedings of the 2016 International Conference on Audio, Language and Image Processing (ICALIP), Shanghai, China, 11-12 July 2016.

12. Zhang, H.-R.; Min, F.; He, X.; Xu, Y.Y. A hybrid recommender system based on user-recommender interaction. Math. Probl. Eng. 2015, 2015, 145636. [CrossRef]

13. Deshpande, M.; Karypis, G. Item-based top-N recommendation algorithms. ACM Trans. Inf. Syst. 2004, 22, 143-177. [CrossRef]

14. Koren, Y.; Bell, R.; Volinsky, C. Matrix factorization techniques for recommender systems. Computer 2009, 8, 30-37. [CrossRef]

15. Hu, Y.; Koren, Y.; Volinsky, C. Collaborative Filtering for Implicit Feedback Datasets. In Proceedings of the Eighth IEEE International Conference on Data Mining, Pisa, Italy, 15-19 December 2009; pp. 263-272.

16. Rendle, S.; Freudenthaler, C.; Gantner, Z.; Schmidt-Thieme, L. BPR: Bayesian personalized ranking from implicit feedback. In Proceedings of the 25th Conference on Uncertainty in Artificial Intelligence, Montreal, QC, Canada, 18-21 June 2009; AUAI Press: Arlington, VA, USA, 2009.

17. He, R.; McAuley, J. VBPR: Visual Bayesian Personalized Ranking from Implicit Feedback. In Proceedings of the AAAI, Phoenix, AZ, USA, 12-17 February 2016; pp. 144-150.

18. Li, D.; Zhao, G.; Wang, Z.; Ma, W.; Liu, Y. A Method of Purchase Prediction Based on User Behavior Log. In Proceedings of the IEEE International Conference on Data Mining Workshop, Atlantic City, NJ, USA, 14-17 November 2016; pp. 1031-1039.

19. Jia, Y.; Zhang, C.; Lu, Q.; Wang, P. Users' brands preference based on SVD++ in recommender systems. In Proceedings of the 2014 IEEE Workshop on Advanced Research and Technology in Industry Applications (WARTIA), Ottawa, ON, Canada, 29-30 September 2014; pp. 1175-1178.

20. Barkan, O.; Koenigstein, N. Item2vec: Neural item embedding for collaborative filtering. In Proceedings of the IEEE 26th International Workshop on Machine Learning for Signal Processing (MLSP), Vietri sul Mare, Italy, 13-16 September 2016.

21. Ozsoy, M.G. From word embeddings to item recommendation. arXiv 2016, arXiv:1601.01356.

22. Mikolov, T.; Chen, K.; Corrado, G.; Dean, J. Efficient Estimation of Word Representations in Vector Space. arXiv 2013, arXiv:1301.3781.

23. Mikolov, T.; Sutskever, I.; Chen, K.; Corrado, G.S.; Dean, J. Distributed Representations of Words and Phrases and their Compositionality. In Proceedings of the Advances in Neural Information Processing Systems, Lake Tahoe, NV, USA, 5-10 December 2013; Volume 26, pp. 3111-3119.

24. Krishnamurthy, B.; Puri, N.; Goel, R. Learning vector-space representations of items for recommendations using word embedding models. Procedia Comput. Sci. 2016, 80, 2205-2210. [CrossRef]

(C) 2018 by the authors. Licensee MDPI, Basel, Switzerland. This article is an open access article distributed under the terms and conditions of the Creative Commons Attribution (CC BY) license (http:/ / creativecommons.org/licenses/by/4.0/). 